\title{
Numerical Performance of Higher-Order Semicompact Scheme for Arbitrary Triangular Cavity Flow
}

\author{
Xiaofeng Wang ${ }^{1,2}$ and Dongyang Shi ${ }^{1}$ \\ ${ }^{1}$ School of Mathematics and Statistics, Zhengzhou University, Zhengzhou 450001, China \\ ${ }^{2}$ School of Mathematical Sciences, Henan Institute of Science and Technology, Xinxiang 453003, China \\ Correspondence should be addressed to Xiaofeng Wang; wangxiaofeng1166@163.com
}

Received 9 June 2013; Revised 13 November 2013; Accepted 2 December 2013; Published 4 February 2014

Academic Editor: Song Cen

Copyright (c) $2014 \mathrm{X}$. Wang and D. Shi. This is an open access article distributed under the Creative Commons Attribution License, which permits unrestricted use, distribution, and reproduction in any medium, provided the original work is properly cited.

\begin{abstract}
An efficient fourth-order semicompact finite difference scheme has been developed to solve steady incompressible Navier-Stokes $(\mathrm{N}-\mathrm{S})$ equations in stream function and vorticity formulation in a triangular cavity of arbitrary geometry. The governing equations are transformed into curvilinear coordinates by a simple linear transformation to handle the nonregular geometry of the problem. The main feature of the new higher-order semicompact scheme is that it can calculate a triangle flow with arbitrary shape for high Reynolds numbers. It is found that the solutions obtained with the present scheme are in good agreement with the analytical results or with the existing results depending on the availability.
\end{abstract}

\section{Introduction}

Numerical simulations for the solution of steady incompressible viscous flow within a driven cavity are a complex and significant topic, and a lot of researchers contributed to this subject [1-4]. Although there are still some minor discrepancies in the results, the square cavity flow has been essentially characterized [5]. As pointed out in McQuain et al. [6], the results for the square cavity may not be applied to other important geometries such as a triangular cavity. Also the latter shapes are more common in practice.

The problem under consideration is the steady motion of an incompressible viscous flow in a triangular cavity of arbitrary geometry. This flow was studied numerically by McQuain et al. [6], Jyotsna and Vanka [7], Li and Tang [8], and Gaskell et al. [9]. McQuain et al. [6] applied the Batchelor's mean square law to triangular cavity flow and analytically obtained the inviscid core vorticity for infinite Reynolds number. Recent calculations of the steady problem in an equilateral triangular cavity have been given in Ribbens et al. [10] for $\mathrm{Re} \leq 500$. Erturk and Gokcol [11] have presented high accurate, fine grid solutions of 2D steady incompressible flow in triangle cavities. The governing equations are solved up to very low residuals at various Reynolds numbers. The results of these studies, however, show some discrepancies.
Furthermore, the scheme in [11] is only second-order spatial accuracy. This constitutes the main motivation of this study.

The main object of this study is the development of an accurate and efficient scheme for solving the N-S problems in triangular geometries. In the present paper, we have attempted an efficient fourth-order semicompact scheme (compactness of the scheme is relaxed for few terms of the governing equations) for stream function vorticity form of incompressible Navier-Stokes equations in curvilinear coordinates inside a triangular cavity. A geometric transformation handling triangles of arbitrary shape is also presented. The developed equations are solved numerically subject to appropriate boundary conditions by a fourth-order accurate finite difference method. Overall, besides opening up new possibilities, the method may be considered an efficient one for computation of flow for this physical configuration and the results thus obtained are not only useful for engineers in the process design; they also supplement the existing literature.

\section{Governing Equations}

A triangular driven cavity of general shape is given by locating its three vertices at $\widehat{A}\left(x_{a}, h\right), \widehat{B}\left(x_{b}, h\right)$, and $\widehat{C}\left(x_{c}, y_{c}\right)$, with the 
upper side moving to the right via a constant velocity $U_{0}$; see Figure 1(a). The 2D steady incompressible flow inside a triangular cavity is governed by the N-S equations. We consider the N-S equations in streamfunction and vorticity formulation in Cartesian coordinates, such that [5]

$$
\begin{gathered}
\nabla_{x y}^{2} \psi=-\omega, \\
\nabla_{x y}^{2} \omega=\operatorname{Re}\left(\mathbf{U} \cdot \nabla_{x y} \omega\right),
\end{gathered}
$$

where

$$
\begin{gathered}
\nabla_{x y}^{2}=\frac{\partial^{2}}{\partial x^{2}}+\frac{\partial^{2}}{\partial y^{2}}, \quad \nabla_{x y}=\left(\frac{\partial}{\partial x}, \frac{\partial}{\partial y}\right), \\
\mathbf{U}=(u, v), \quad u=\frac{\partial \psi}{\partial y}, \quad v=-\frac{\partial \psi}{\partial x}, \quad \omega=\frac{\partial v}{\partial x}-\frac{\partial u}{\partial y},
\end{gathered}
$$

where $\psi$ is the streamfunction, $\omega$ is the vorticity, $u$ and $v$ are the components of the velocity in $x$ - and $y$-directions, respectively, and Re is Reynolds number. We note that these equations are non dimensional and a length scale of $\left(h-c_{y}\right) / 3$ and a velocity scale of $U_{0}$, that is, the velocity of the lid, are used to nondimensionalize the parameters and Reynolds number is defined accordingly.

By a simple linear transformation

$$
\begin{gathered}
\xi=\frac{x-x_{a}}{x_{b}-x_{a}}+\frac{\left(x_{a}-x_{c}\right)(y-h)}{\left(x_{b}-x_{a}\right)\left(y_{c}-h\right)}, \\
\eta=\frac{\sqrt{\left(y_{c}-h\right)^{2}+\left(x_{c}-x_{a}\right)^{2}}}{\left(x_{b}-x_{a}\right)\left(y_{c}-h\right)}(y-h),
\end{gathered}
$$

the triangle $\triangle \widehat{A} \widehat{B} \widehat{C}$ is transformed to a rightangled triangle with vertices $A(0,0), B(1,0)$, and $C\left(0, \sqrt{\left(y_{c}-h\right)^{2}+\left(x_{c}-x_{a}\right)^{2}} /\left(x_{b}-x_{a}\right)\right)$; see Figure 1(b). From these relations, we can calculate the transformation metrics as follows:

$$
\begin{aligned}
& \frac{\partial \xi}{\partial x}=\frac{1}{x_{b}-x_{a}}, \quad \frac{\partial \xi}{\partial y}=\frac{x_{a}-x_{c}}{\left(x_{b}-x_{a}\right)\left(y_{c}-h\right)}, \\
& \frac{\partial \eta}{\partial x}=0, \quad \frac{\partial \eta}{\partial y}=\frac{\sqrt{\left(y_{c}-h\right)^{2}+\left(x_{c}-x_{a}\right)^{2}}}{\left(x_{b}-x_{a}\right)\left(y_{c}-h\right)},
\end{aligned}
$$

and also

$$
\frac{\partial^{2} \xi}{\partial x^{2}}=\frac{\partial^{2} \xi}{\partial y^{2}}=\frac{\partial^{2} \eta}{\partial x^{2}}=\frac{\partial^{2} \eta}{\partial y^{2}}=0
$$

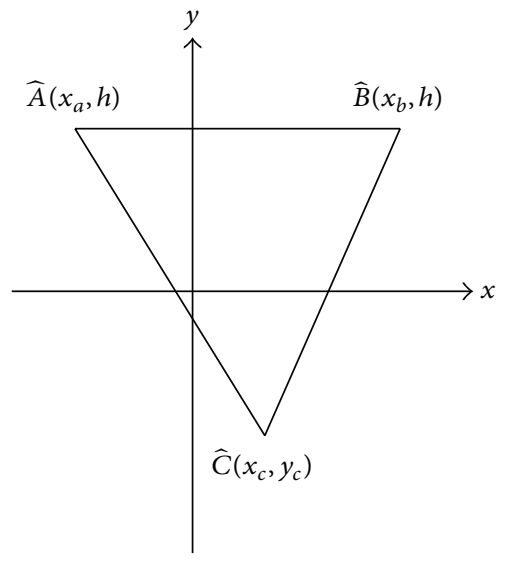

(a)

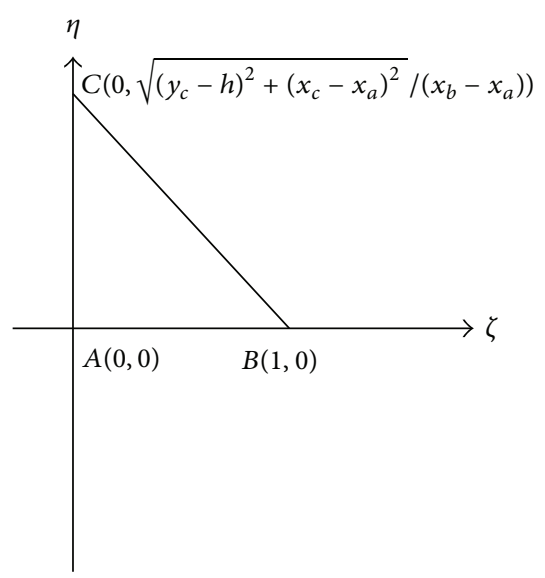

(b)

FIGURE 1: Geometric transformation of the triangular cavity: Physical domain (a) and Computational domain (b).

Using the chain rule, the governing N-S equations (1) in general curvilinear coordinates are as follows:

$$
\begin{gathered}
\left(\left(\frac{\partial \xi}{\partial x}\right)^{2}+\left(\frac{\partial \xi}{\partial y}\right)^{2}\right) \frac{\partial^{2} \psi}{\partial \xi^{2}}+\left(\left(\frac{\partial \eta}{\partial x}\right)^{2}+\left(\frac{\partial \eta}{\partial y}\right)^{2}\right) \frac{\partial^{2} \psi}{\partial \eta^{2}} \\
+\left(\frac{\partial^{2} \xi}{\partial x^{2}}+\frac{\partial^{2} \xi}{\partial y^{2}}\right) \frac{\partial \psi}{\partial \xi}+\left(\frac{\partial^{2} \eta}{\partial x^{2}}+\frac{\partial^{2} \eta}{\partial y^{2}}\right) \frac{\partial \psi}{\partial \eta} \\
+2\left(\frac{\partial \xi}{\partial x} \frac{\partial \eta}{\partial x}+\frac{\partial \xi}{\partial y} \frac{\partial \eta}{\partial y}\right) \frac{\partial^{2} \psi}{\partial \xi \partial \eta}=-\omega \\
\left(\left(\frac{\partial \xi}{\partial x}\right)^{2}+\left(\frac{\partial \xi}{\partial y}\right)^{2}\right) \frac{\partial^{2} \omega}{\partial \xi^{2}}+\left(\left(\frac{\partial \eta}{\partial x}\right)^{2}+\left(\frac{\partial \eta}{\partial y}\right)^{2}\right) \frac{\partial^{2} \omega}{\partial \eta^{2}} \\
+\left(\frac{\partial^{2} \xi}{\partial x^{2}}+\frac{\partial^{2} \xi}{\partial y^{2}}\right) \frac{\partial \omega}{\partial \xi}+\left(\frac{\partial^{2} \eta}{\partial x^{2}}+\frac{\partial^{2} \eta}{\partial y^{2}}\right) \frac{\partial \omega}{\partial \eta} \\
+2\left(\frac{\partial \xi}{\partial x} \frac{\partial \eta}{\partial x}+\frac{\partial \xi}{\partial y} \frac{\partial \eta}{\partial y}\right) \frac{\partial^{2} \omega}{\partial \xi \partial \eta}=\Omega,
\end{gathered}
$$

where

$$
\Omega=\operatorname{Re} \frac{\partial \xi}{\partial x} \frac{\partial \eta}{\partial y}\left(\frac{\partial \psi}{\partial \eta} \frac{\partial \omega}{\partial \xi}-\frac{\partial \psi}{\partial \xi} \frac{\partial \omega}{\partial \eta}\right)
$$


Substituting for the transformation metrics, we obtain the equations that govern the flow in a triangular cavity shown in Figure 1(b) as follows:

$$
\begin{gathered}
\nabla_{\xi \eta}^{2} \psi=-\omega \\
\nabla_{\xi \eta}^{2} \omega=J\left(\frac{\partial \psi}{\partial \eta} \frac{\partial \omega}{\partial \xi}-\frac{\partial \psi}{\partial \xi} \frac{\partial \omega}{\partial \eta}\right)
\end{gathered}
$$

where

$$
\begin{gathered}
\nabla_{\xi \eta}^{2}=\alpha \frac{\partial^{2}}{\partial \xi^{2}}+\beta \frac{\partial^{2}}{\partial \eta^{2}}+\gamma \frac{\partial^{2}}{\partial \xi \partial \eta}, \quad \alpha=\left(\frac{\partial \xi}{\partial x}\right)^{2}+\left(\frac{\partial \xi}{\partial y}\right)^{2} \\
\beta=\left(\frac{\partial \eta}{\partial y}\right)^{2}, \quad \gamma=2 \frac{\partial \xi}{\partial y} \frac{\partial \eta}{\partial y}, \quad J=\operatorname{Re} \frac{\partial \xi}{\partial x} \frac{\partial \eta}{\partial y}
\end{gathered}
$$

and we obtain

$$
\alpha=\beta, \quad u=\frac{\partial \psi}{\partial \xi} \frac{\partial \xi}{\partial y}+\frac{\partial \psi}{\partial \eta} \frac{\partial \eta}{\partial y}, \quad v=-\frac{\partial \psi}{\partial \xi} \frac{\partial \xi}{\partial x} .
$$

The boundary conditions in $x y$-plane are follows [11]:

$$
\psi=0 \text { on all three sides, }
$$

$$
(u, v) \cdot \tau= \begin{cases}1 & \text { on the top side } \\ 0 & \text { on the other two sides }\end{cases}
$$

$$
(u, v) \cdot n=0 \text { on all three sides, }
$$

where $n$ is the outside normal unit vector and $\tau$ is the tangential unit vector in clockwise direction. In the $\xi \eta$-plane condition (11) remains the same form, while (13)-(14) need to be converted as follows. On the top side $\widehat{A} \widehat{B}$, substituting (11) into (13)-(14), with $\tau=(1,0)$ and $n=(0,1)$ yields

$$
\begin{aligned}
& \frac{\partial \psi}{\partial \xi}=0 \\
& \frac{\partial \psi}{\partial \eta}=\frac{\partial y}{\partial \eta}=\frac{\left(x_{b}-x_{a}\right)\left(y_{c}-h\right)}{\sqrt{\left(y_{c}-h\right)^{2}+\left(x_{c}-x_{a}\right)^{2}}} \text { on side } A B .
\end{aligned}
$$

On side $\widehat{B} \widehat{C}$ we have

$$
\begin{aligned}
& \tau=\frac{\left(x_{c}-x_{b}, y_{c}-h\right)}{\sqrt{\left(x_{c}-x_{b}\right)^{2}+\left(y_{c}-h\right)^{2}}}, \\
& n=\frac{\left(h-y_{c}, x_{c}-x_{b}\right)}{\sqrt{\left(x_{c}-x_{b}\right)^{2}+\left(y_{c}-h\right)^{2}}} .
\end{aligned}
$$

Combining this with (11) we obtain

$$
\frac{\partial \psi}{\partial \xi}=0, \quad \frac{\partial \psi}{\partial \eta}=0 \quad \text { on side } B C .
$$

Similarly on side $\widehat{C} \widehat{A}$ by using

$$
\begin{aligned}
& \tau=\frac{\left(x_{a}-x_{c}, h-y_{c}\right)}{\sqrt{\left(x_{a}-x_{c}\right)^{2}+\left(y_{c}-h\right)^{2}}}, \\
& n=\frac{\left(y_{c}-h, x_{a}-x_{c}\right)}{\sqrt{\left(x_{a}-x_{c}\right)^{2}+\left(y_{c}-h\right)^{2}}} .
\end{aligned}
$$

we obtain

$$
\frac{\partial \psi}{\partial \xi}=0, \quad \frac{\partial \psi}{\partial \eta}=0 \quad \text { on side } C A
$$

\section{Higher-Order Semicompact Scheme}

3.1. Discretization of the Stream Function Equation. It is known that the higher-order compact approximation for any Poisson type of operator acting on $f$ is given by $[12,13]$

$$
\begin{gathered}
D_{x}^{2}\left(1+\frac{\Delta h^{2}}{12} D_{y}^{2}\right) f+D_{y}^{2}\left(1+\frac{\Delta h^{2}}{12} D_{x}^{2}\right) f \\
=\left(1+\frac{\Delta h^{2}}{12}\left(D_{x}^{2}+D_{y}^{2}\right)\right) g,
\end{gathered}
$$

where $g$ is any known function, $\Delta h$ is the constant step length in $x$ and $y$ directions, and

$$
\begin{aligned}
D_{x}^{2} f(x, y)=\frac{1}{\Delta h}( & f(x-\Delta h, y)-2 f(x, y) \\
+ & f(x+\Delta h, y)), \\
D_{y}^{2} f(x, y)=\frac{1}{\Delta h}( & f(x, y-\Delta h)-2 f(x, y) \\
+ & f(x, y+\Delta h)) .
\end{aligned}
$$

If we define

$$
\left(1+\frac{\Delta h^{2}}{12}\left(D_{\xi}^{2}+D_{\eta}^{2}\right)\right) \omega=\widetilde{\omega},
$$

and then approximate the stream function (8) in the form of (25) we get

$$
\begin{aligned}
& \alpha D_{\xi}^{2}\left(1+\frac{\Delta h^{2}}{12} D_{\eta}^{2}\right) \psi+\beta D_{\eta}^{2}\left(1+\frac{\Delta h^{2}}{12} D_{\xi}^{2}\right) \psi+\widetilde{\gamma} \psi \\
& =-\widetilde{\omega}+O\left(\Delta h^{4}\right)
\end{aligned}
$$

where

$$
\begin{gathered}
\tilde{\gamma}=\gamma D_{\xi \eta}^{2}\left(1-\frac{\Delta h^{2}}{12}\left(D_{\xi}^{2}+D_{\eta}^{2}\right)\right), \\
D_{\xi} f(\xi, \eta)=\frac{1}{2 \Delta h}(f(\xi-\Delta h, \eta)-f(\xi+\Delta h, \eta)), \\
D_{\eta} f(\xi, \eta)=\frac{1}{2 \Delta h}(f(\xi, \eta-\Delta h)-f(\xi, \eta+\Delta h)) .
\end{gathered}
$$

A 2D finite difference scheme is compact if it only involves at most the 8 nearest neighboring grid points (of the center point) in the approximation formula [14]. Then in (23), first and second terms on the left side of the equation can be discretized on the compact nine point stencil as in any other compact scheme. However, discretization of the third term $\tilde{\gamma} \psi$ needs some special attention. We refer this term as TERM 1 and the discretization of this term is considered after the discussion of the vorticity equation. 


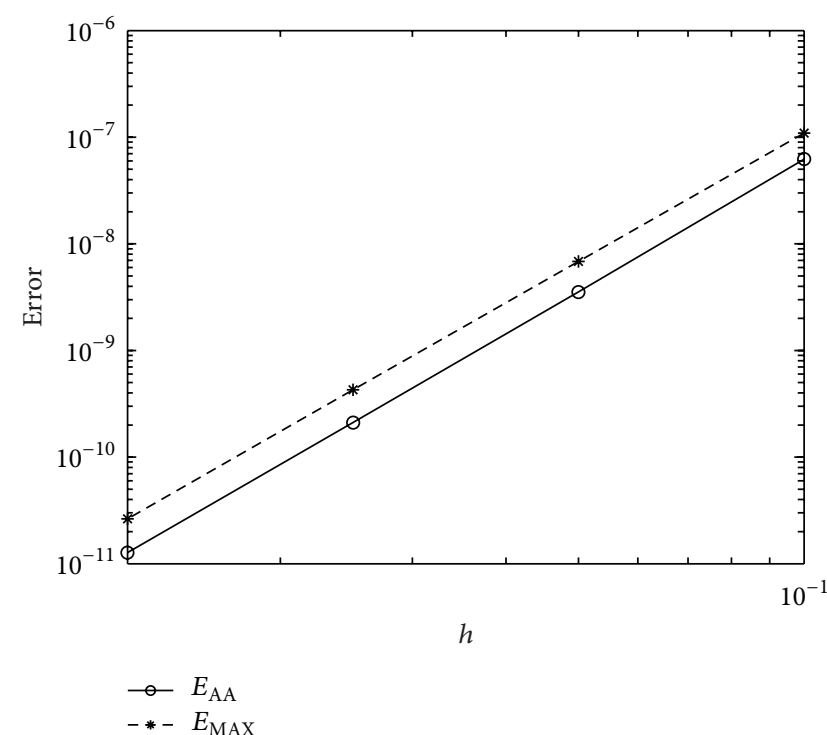

(a)

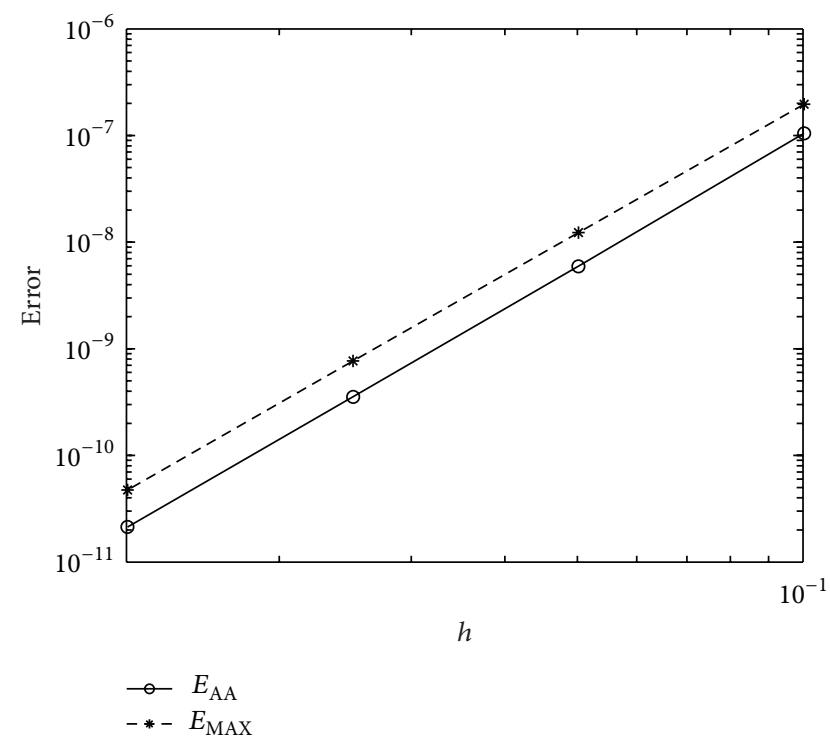

(b)

FIGURE 2: The average absolute error $E_{A A}$ and the maximum norm error $E_{\mathrm{MAX}}$ in streamfunction and vorticity variables with respect to grid spacing. (a) streamfunction and (b) vorticity.

TABLE 1: Properties of the center of the primary eddy, located at $(x, y)$ with streamfunction value and vorticity for equilateral triangle.

\begin{tabular}{|c|c|c|c|c|}
\hline$\overline{\operatorname{Re}}$ & Source & $\psi$ & $\omega$ & $(x, y)$ \\
\hline \multirow{5}{*}{1} & Present & -0.2348 & -1.4335 & $(0.0271,0.4844)$ \\
\hline & Li and Tang [8] & -0.2340 & -1.3990 & $(0.0000,0.4750)$ \\
\hline & Erturk and Gokcol [11] & -0.2329 & -1.3788 & $(0.0101,0.4668)$ \\
\hline & Gaskell et al. [9] & -0.2320 & -1.3750 & $(0.0100,0.4670)$ \\
\hline & McQuain et al. [6] & -0.2330 & -1.3630 & $(0.0170,0.4600)$ \\
\hline \multirow{5}{*}{50} & Present & -0.2377 & -1.4533 & $(0.3248,0.4375)$ \\
\hline & Li and Tang [8] & -0.2350 & -1.4380 & $(0.3680,0.4380)$ \\
\hline & Erturk and Gokcol [11] & -0.2369 & -1.4689 & $(0.3484,0.4434)$ \\
\hline & Gaskell et al. [9] & -0.2360 & -1.4690 & $(0.3500,0.4420)$ \\
\hline & McQuain et al. [6] & -0.2370 & -1.4640 & $(0.3460,0.4450)$ \\
\hline \multirow{5}{*}{100} & Present & -0.2489 & -1.3795 & $(0.3291,0.3700)$ \\
\hline & $\mathrm{Li}$ and Tang $[8]$ & -0.2440 & -1.2640 & $(0.3680,0.3630)$ \\
\hline & Erturk and Gokcol [11] & -0.2482 & -1.3669 & $(0.3315,0.3555)$ \\
\hline & Gaskell et al. [9] & -0.2490 & -1.3650 & $(0.3330,0.3560)$ \\
\hline & McQuain et al. [6] & -0.2470 & -1.3730 & $(0.3290,0.3550)$ \\
\hline \multirow{5}{*}{350} & Present & -0.2663 & -1.2388 & $(0.1624,0.2500)$ \\
\hline & Li and Tang [8] & -0.2740 & -1.1530 & $(0.1520,0.2130)$ \\
\hline & Erturk and Gokcol [11] & -0.2724 & -1.1985 & $(0.1556,0.2383)$ \\
\hline & Gaskell et al. [9] & -0.2740 & -1.2010 & $(0.1490,0.2340)$ \\
\hline & McQuain et al. [6] & -0.2680 & -1.2320 & $(0.1730,0.2650)$ \\
\hline \multirow{5}{*}{500} & Present & -0.2748 & -1.1992 & $(0.1299,0.2350)$ \\
\hline & Li and Tang [8] & -0.2780 & -1.1240 & $(0.1080,0.2130)$ \\
\hline & Erturk and Gokcol [11] & -0.2774 & -1.1791 & $(0.1319,0.2207)$ \\
\hline & Gaskell et al. [9] & -0.2810 & -1.1870 & $(0.1280,0.2170)$ \\
\hline & McQuain et al. [6] & -0.2690 & -1.2500 & $(0.1730,0.2650)$ \\
\hline \multirow{3}{*}{1000} & Present & -0.2902 & -1.2067 & $(0.0866,0.2200)$ \\
\hline & Li and Tang [8] & -0.2790 & -1.0480 & $(0.1080,0.1380)$ \\
\hline & Erturk and Gokcol [11] & -0.2844 & -1.1629 & $(0.1116,0.1973)$ \\
\hline \multirow{3}{*}{1500} & Present & -0.2962 & -1.2282 & $(0.0664,0.2250)$ \\
\hline & Li and Tang [8] & -0.2770 & -0.9980 & $(0.1080,0.1380)$ \\
\hline & Erturk and Gokcol [11] & -0.2873 & -1.1639 & $(0.1015,0.1914)$ \\
\hline \multirow{3}{*}{2000} & Present & -0.2951 & -1.1827 & $(0.0693,0.2029)$ \\
\hline & Li and Tang [8] & - & - & - \\
\hline & Erturk and Gokcol [11] & - & - & - \\
\hline
\end{tabular}




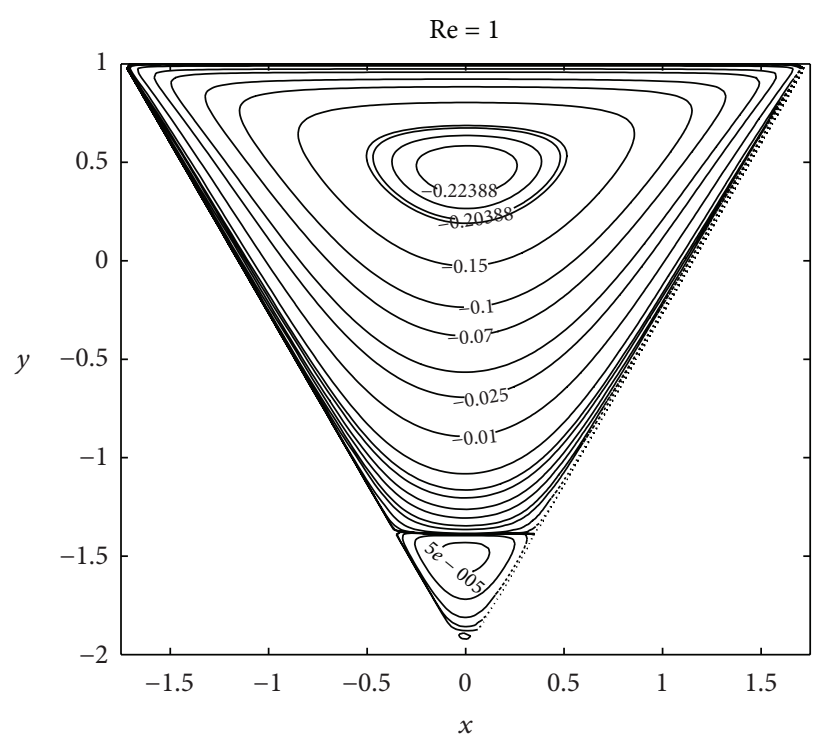

(a)

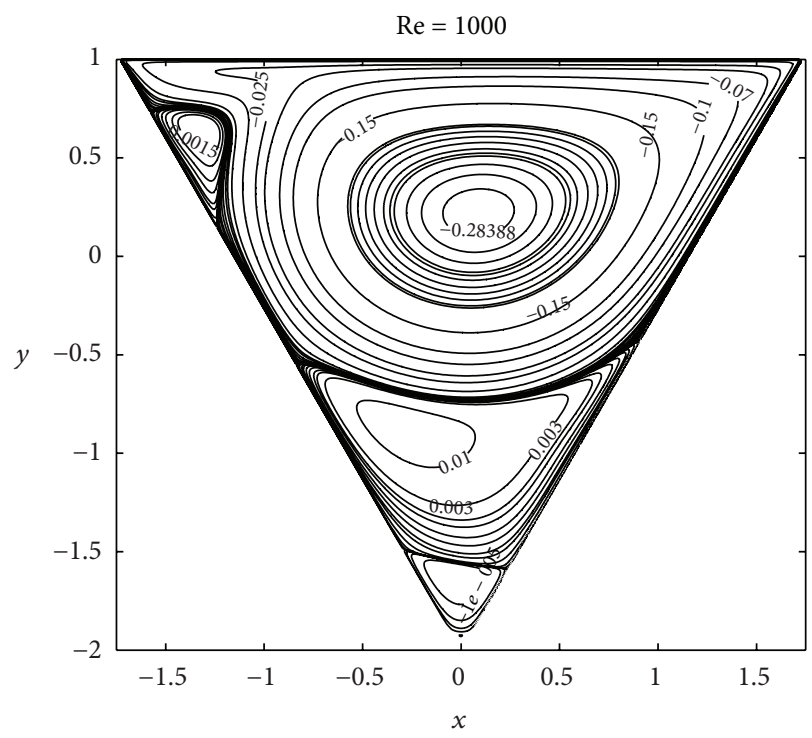

(c)

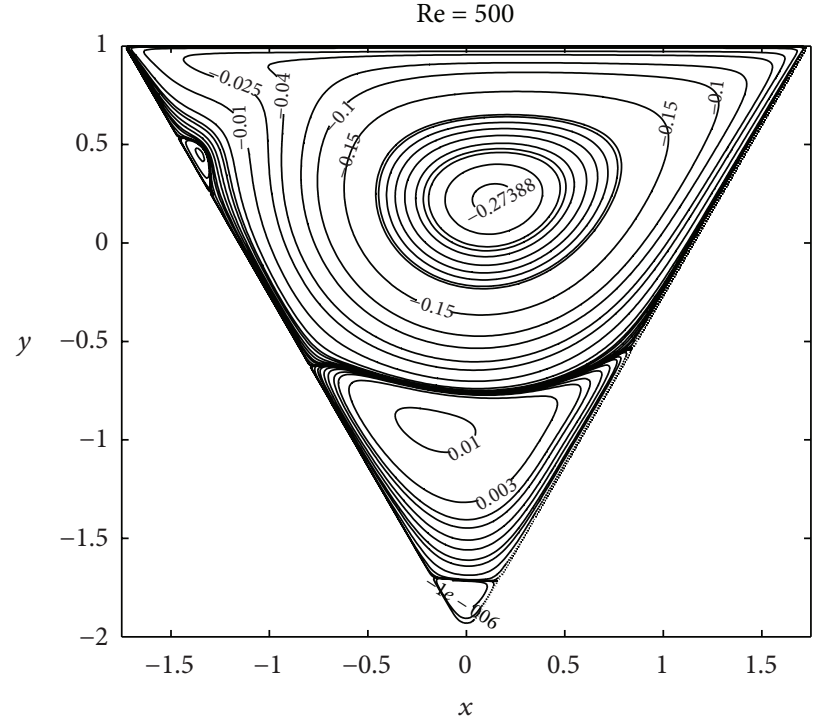

(b)

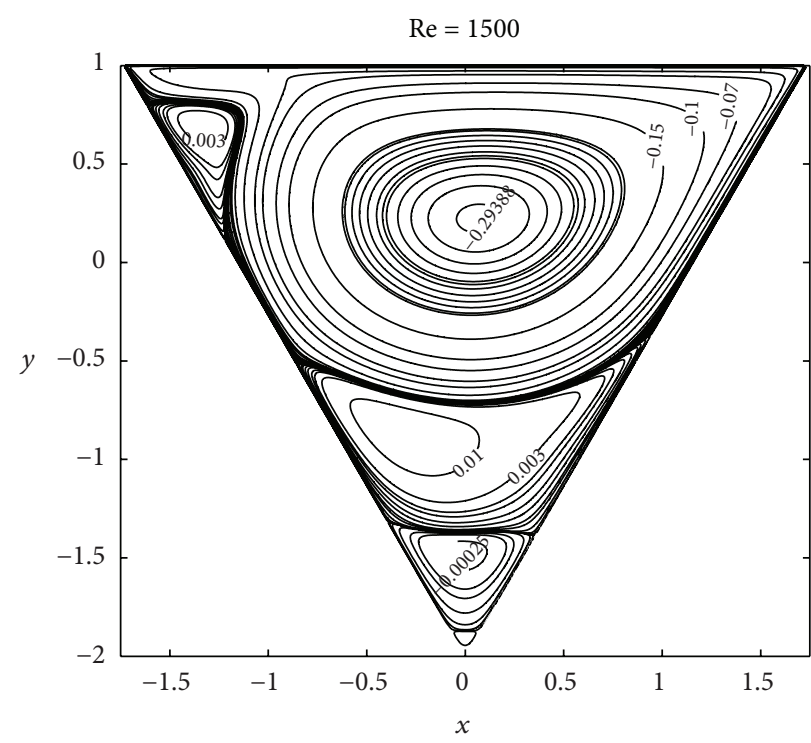

(d)

FIGURE 3: Streamline contours of triangular cavity flow at various Reynolds numbers.

3.2. Discretization of the Vorticity Equation. Equation (9) with fourth-order approximation can be written as

$$
\begin{aligned}
\alpha D_{\xi}^{2}(1 & \left.+\frac{\Delta h^{2}}{12} D_{\eta}^{2}\right) \omega+\beta D_{\eta}^{2}\left(1+\frac{\Delta h^{2}}{12} D_{\xi}^{2}\right) \omega+\tilde{\gamma} \omega \\
= & J\left(1+\frac{\Delta h^{2}}{12}\left(D_{\xi}^{2}+D_{\eta}^{2}\right)\right)\left(D_{\eta} \psi D_{\xi} \omega-D_{\xi} \psi D_{\eta} \omega\right) \\
& +O\left(\Delta h^{4}\right) .
\end{aligned}
$$

The first and second terms on the left hand side of (25) can be discretized within the compact stencil. We call the third term $\tilde{\gamma} \omega$ as TERM2 which needs a larger stencil to approximate to fourth-order. The term on the right hand side of (25) contains

$$
\begin{aligned}
\left(D_{\xi}^{2}+\right. & \left.D_{\eta}^{2}\right)\left(D_{\eta} \psi D_{\xi} \omega-D_{\xi} \psi D_{\eta} \omega\right) \\
= & 2 D_{\xi \eta}^{2} \omega\left(D_{\eta}^{2}-D_{\xi}^{2}\right) \psi+2 D_{\xi \eta}^{2} \psi\left(D_{\xi}^{2}-D_{\eta}^{2}\right) \omega \\
& -D_{\xi}\left(D_{\xi}^{2}+D_{\eta}^{2}\right) \psi D_{\eta} \omega-D_{\xi} \psi D_{\eta}\left(D_{\xi}^{2}+D_{\eta}^{2}\right) \omega \\
& +D_{\eta}\left(D_{\xi}^{2}+D_{\eta}^{2}\right) \psi D_{\xi} \omega+D_{\eta} \psi D_{\xi}\left(D_{\xi}^{2}+D_{\eta}^{2}\right) \omega .
\end{aligned}
$$

The first two terms on the right hand side of (26) again can be discretized within the compact stencil to the fourth-order; 


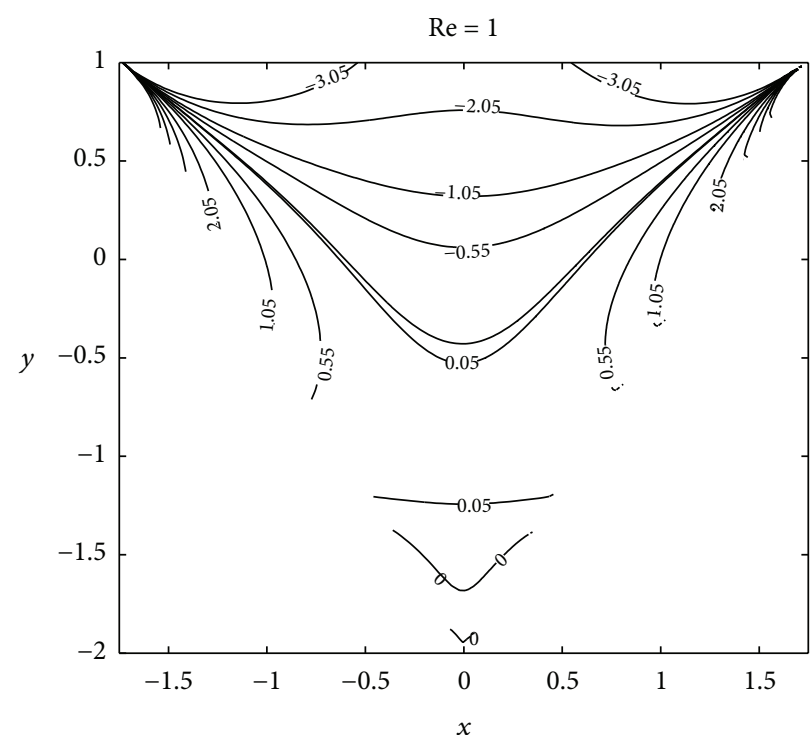

(a)

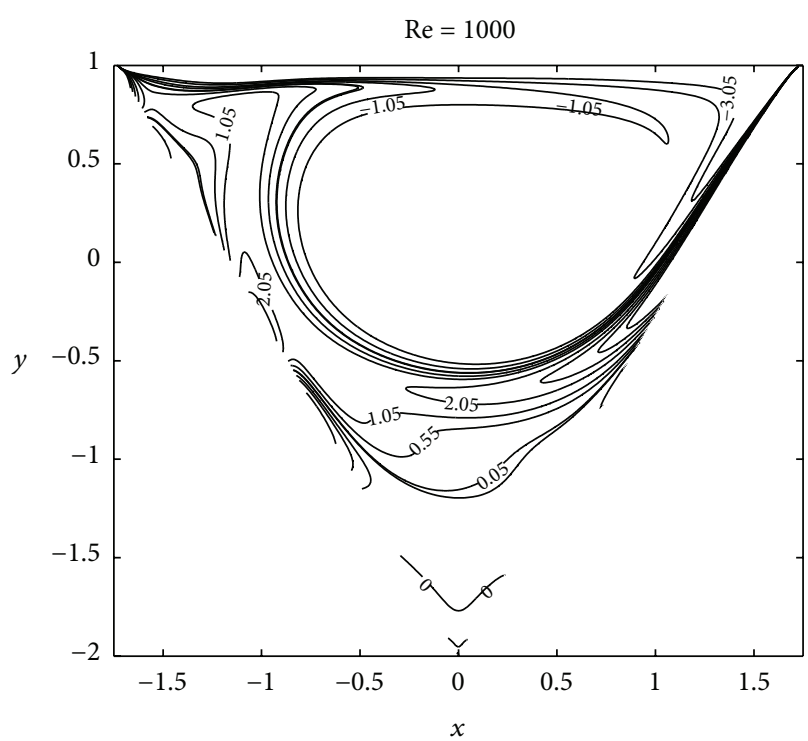

(c)

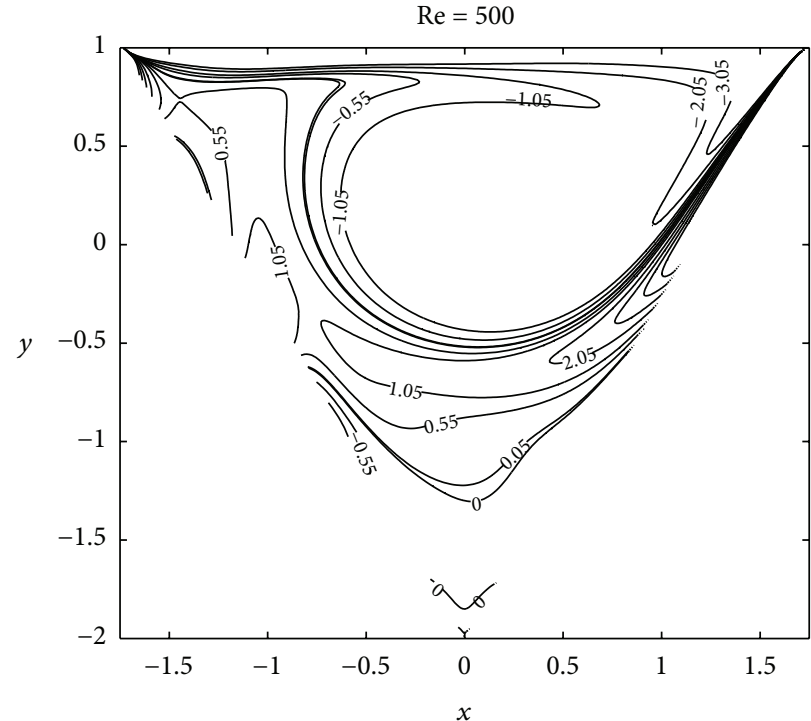

(b)

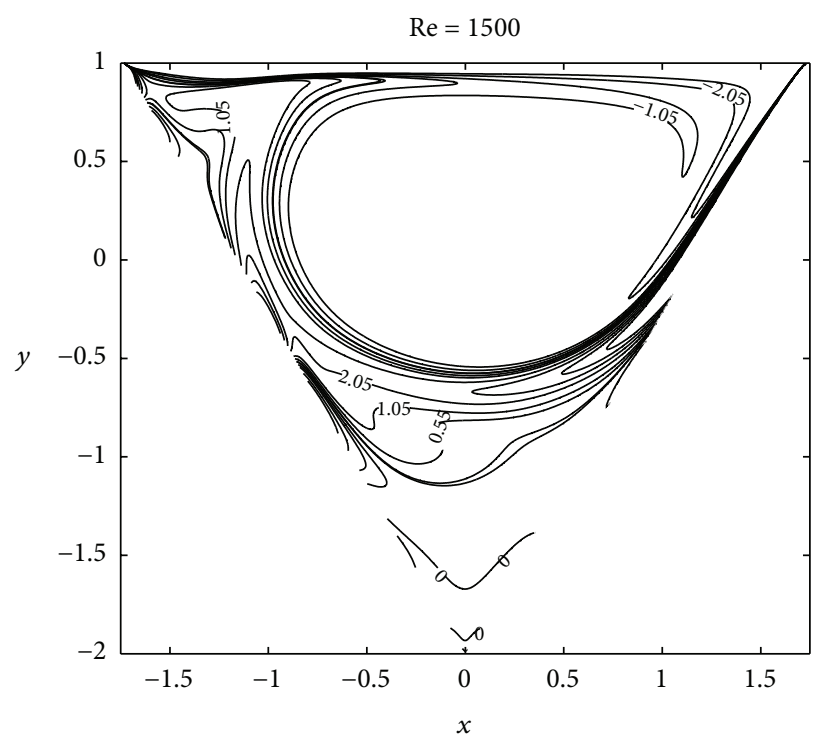

(d)

FIgURE 4: Vorticity contours of triangular cavity flow at various Reynolds numbers.

however, the last four terms, call them as TERM3, TERM4, TERM5, and TERM6, need larger stencil.

3.3. Numerical Treatment of the Terms TERM1-TERM6. We obtain from (8) that

$$
\frac{\partial^{2} \psi}{\partial \xi^{2}}+\frac{\partial^{2} \psi}{\partial \eta^{2}}=-\frac{\omega}{\alpha}-\frac{\gamma}{\alpha} \frac{\partial^{2} \psi}{\partial \xi \partial \eta}
$$

Therefore,

$$
D_{\xi \eta}^{2}\left(D_{\xi}^{2}+D_{\eta}^{2}\right) \psi=-\frac{1}{\alpha} D_{\xi \eta}^{2} \omega-\frac{\gamma}{\alpha} D_{\xi \xi \eta \eta}^{4} \psi
$$

Similarly, from (9) we obtain

$$
\begin{aligned}
& D_{\xi \eta}^{2}\left(D_{\xi}^{2}+D_{\eta}^{2}\right) \omega \\
&=-\frac{\gamma}{\alpha} D_{\xi \xi \eta \eta}^{4} \omega+\frac{J}{\alpha}\left(D_{\xi \eta \eta}^{3} \psi D_{\xi} \omega+D_{\eta} \psi D_{\xi \xi \eta}^{3} \omega\right. \\
&\left.\quad+D_{\eta}^{2} \psi D_{\xi}^{2} \omega\right) \\
& \quad-\frac{J}{\alpha}\left(D_{\xi \xi \eta}^{3} \psi D_{\eta} \omega+D_{\xi} \psi D_{\xi \eta \eta}^{3} \omega+D_{\xi}^{2} \psi D_{\eta}^{2} \omega\right) .
\end{aligned}
$$

In (29), we have third derivatives such as $D_{\xi}\left(D_{\xi}^{2}+D_{\eta}^{2}\right) \psi$ and $D_{\eta}\left(D_{\xi}^{2}+D_{\eta}^{2}\right) \omega$ which present some problems to the fourthorder compact formulation. In order to find an expression 


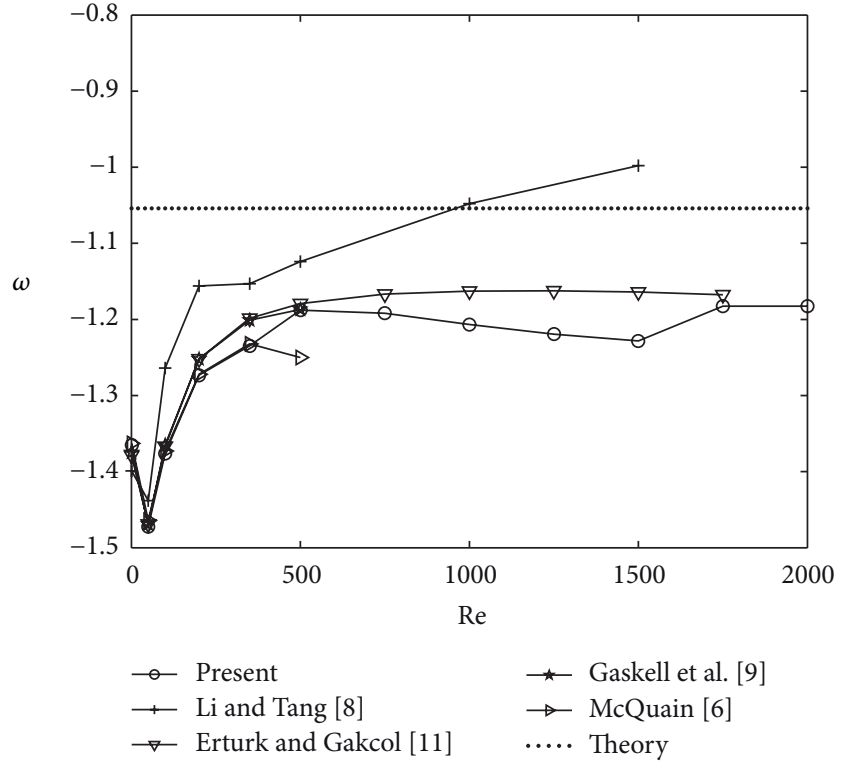

(a)

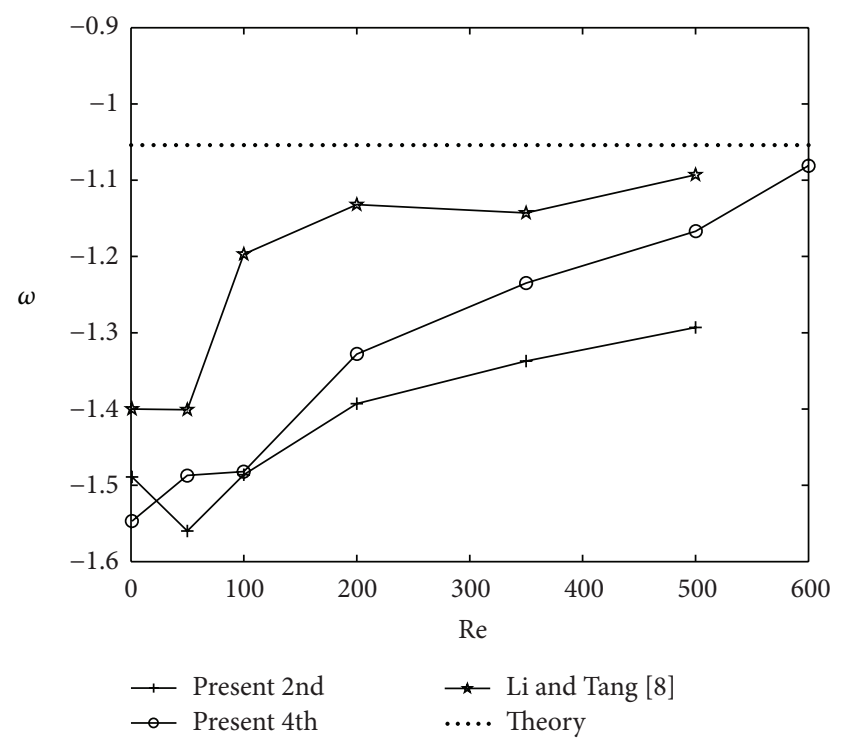

(b)

FIGURE 5: Comparison of the vorticity at the center of the primary eddy in the equilateral triangular cavity (a) and right-oriented triangular cavity (b).

for these derivatives, we use (8)-(9) to obtain the following equations:

$$
\begin{gathered}
D_{\kappa}\left(D_{\xi}^{2}+D_{\eta}^{2}\right) \psi=-\frac{1}{\alpha} D_{\kappa} \omega-\frac{\gamma}{\alpha} D_{\xi \kappa \eta}^{3} \psi, \quad \kappa=\xi, \eta, \\
D_{\kappa}\left(D_{\xi}^{2}+D_{\eta}^{2}\right) \omega=-\frac{\gamma}{\alpha} D_{\xi \kappa \eta}^{3} \omega+\frac{J}{\alpha}\left(D_{\xi} \omega D_{\kappa \eta}^{2} \psi+D_{\xi \kappa}^{2} \omega D_{\eta} \psi\right) \\
-\frac{J}{\alpha}\left(D_{\xi \kappa}^{2} \psi D_{\eta} \omega+D_{\xi} \psi D_{\kappa \eta}^{2} \omega\right), \\
\kappa=\xi, \eta .
\end{gathered}
$$

3.4. Numerical Boundary Conditions. The numerical implementation of the boundary conditions for $u, v$, and $\psi$ are straightforward. On side $A B$ boundary, we have

$$
\omega_{i, 1}=-\beta \frac{\partial^{2} \psi_{i, 1}}{\partial \eta^{2}}, \quad \alpha \frac{\partial^{2} \omega_{i, 1}}{\partial \xi^{2}}+\beta \frac{\partial^{2} \omega_{i, 1}}{\partial \eta^{2}}+\gamma \frac{\partial^{2} \omega_{i, 1}}{\partial \xi \partial \eta}=J K \frac{\partial \omega_{i, 1}}{\partial \xi},
$$

where $i=2,3, \ldots, N$ and

$$
K=\frac{\partial \psi_{i, 1}}{\partial \eta}=\frac{\left(x_{b}-x_{a}\right)\left(y_{c}-h\right)}{\sqrt{\left(y_{c}-h\right)^{2}+\left(x_{c}-x_{a}\right)^{2}}} .
$$

Employing Taylor series expansion, we get

$$
\begin{aligned}
K=\frac{\partial \psi_{i, 1}}{\partial \eta}= & D_{\eta}^{+} \psi_{i, 1}-\frac{\Delta h}{2} \frac{\partial^{2} \psi_{i, 1}}{\partial \eta^{2}}-\frac{\Delta h^{2}}{6} \frac{\partial^{3} \psi_{i, 1}}{\partial \eta^{3}} \\
& -\frac{\Delta h^{3}}{24} \frac{\partial^{4} \psi_{i, 1}}{\partial \eta^{4}}+O\left(\Delta h^{4}\right),
\end{aligned}
$$

where $D_{\eta}^{+}$is the first-order forward difference operator in the $\eta$-direction and Using (32) in (34), we get the fourth-order accuracy $\mathrm{O}\left(\Delta h^{4}\right)$ expression as follows:

$$
\begin{aligned}
K= & D_{\eta}^{+} \psi_{i, 1}+\frac{\Delta h}{2 \beta} \omega_{i, 1}+\frac{\Delta h^{2}}{6 \beta} D_{\eta}^{+} \omega_{i, 1} \\
& -\frac{\Delta h^{3}}{24 \beta^{2}}\left(J K D_{\xi}^{+}-\alpha D_{\xi}^{2}-\gamma \delta_{\xi \eta}^{2}\right) \omega_{i, 1},
\end{aligned}
$$

where $D_{\xi}^{+}$is the first-order forward difference operator in the $\xi$-direction, $\delta_{\xi \eta}^{2}$ is the first-order cross difference operator, that is

$$
\delta_{\xi \eta}^{2} \omega_{i, 1}=\frac{1}{\Delta h^{2}}\left(\omega_{i, 2}-\omega_{i, 1}-\omega_{i-1,2}+\omega_{i-1,1}\right)+O(\Delta h) .
$$

Substituting (36) into (35), we get

$$
\omega_{i, 1}=\frac{\Theta-(J K \Delta h-\alpha) \omega_{i+1,1}+(\alpha+\gamma) \omega_{i-1,1}+\gamma \omega_{i-1,2}}{\gamma-J K \Delta h+2 \alpha-8 \beta},
$$

where $\Theta=\theta\left(\psi_{i, 2}-K \Delta h\right)+(4 \beta+\gamma) \omega_{i, 2}, \theta=24 \beta^{2} / \Delta h^{2}$.

Similarly on side $A C$ the vorticity is equal to

$$
\begin{aligned}
& \omega_{1, j} \\
& =\frac{\theta \psi_{2, j}+(4 \beta+\gamma) \omega_{2, j}+\alpha \omega_{1, j+1}+(\alpha+\gamma) \omega_{1, j-1}+\gamma \omega_{2, j-1}}{\gamma+2 \alpha-8 \beta},
\end{aligned}
$$




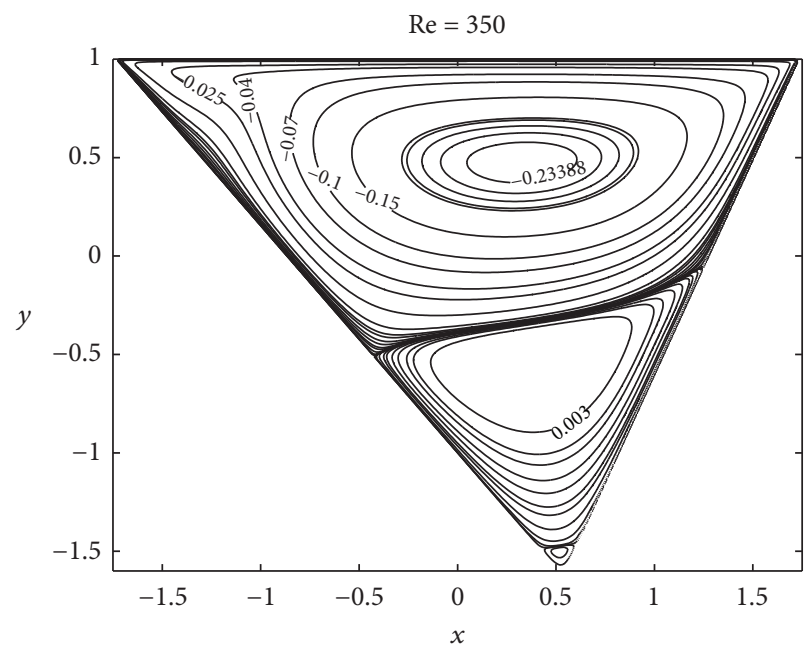

(a)

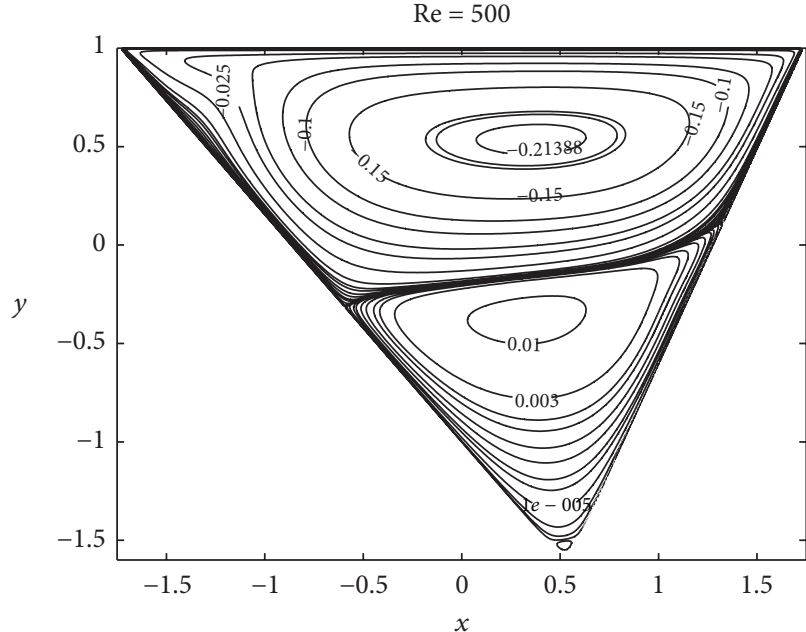

(b)

FIGURE 6: Streamlines distributions for a right-oriented triangular cavity at different Reynolds numbers.

where $j=2,3, \ldots, N$. On side $B C$, it is equal to

$$
\begin{aligned}
& \omega_{i, k} \\
& =\frac{\theta \psi_{i-1, k}+(4 \beta+\gamma) \omega_{i-1, k}+\alpha \omega_{i, k-1}+(\alpha+\gamma) \omega_{i, k+1}+\gamma \omega_{i-1, k+1}}{\gamma+2 \alpha-8 \beta},
\end{aligned}
$$

where $k=N+2-i, i=2,3, \ldots, N$. It is found that the point $(i, k+1)$ is outside the 9-point domain being required and the value of $\omega_{i, k+1}$ is not available; we replace $\omega_{i, k+1}$ by using the following equation:

$$
\begin{aligned}
\omega_{i, k+1}= & 2 \omega_{i, k}-\omega_{i, k-1}+\omega_{i-1, k+1} \\
& -2 \omega_{i-1, k}+\omega_{i-1, k-1}+O\left(\Delta h^{4}\right) .
\end{aligned}
$$

At the three singular corners, the values of vorticity need some special attention in the process of discretization. We use the average of vorticity on the two adjacent points with the singular corners.

\section{Results and Discussion}

Following the numerical procedure described in the previous section, the new schemes (23) and (25) are fourth-order accurate; therefore, they need to be solved in an iterative manner such as SOR iteration with the relaxation parameter $\Phi=2 / 3$. In all of the cases considered, we start the iterations from a homogeneous initial guess and continue until a certain condition of convergence is satisfied. As convergence criteria we decided to use the difference of the $\psi-\omega$ variables between two steps normalized by the previous value of the corresponding variable, such that

$$
\max \left(\left|\psi^{n+1}-\psi^{n}\right|\right)<10^{-8}, \quad \max \left(\left|\omega^{n+1}-\omega^{n}\right|\right)<10^{-8} .
$$

At this convergence level, this would indicate that the variables $\psi$ and $\omega$ are changing less than $0.000001 \%$ of their value between two iterations at every grid point in the mesh. These residuals indicate the degree to which the numerical solution has converged to steady state.

We considered different triangle geometries with different Reynolds numbers. Numerical tests for a variety of triangular geometries have been investigated, with Re up to 2000 for an equilateral cavity and 1500 for scalene cavities. It should be pointed out that Reynolds number is based on $h$, which is consistent with the definition in the case of the equilateral cavity. We solved the flow in this triangle cavity at various Reynolds numbers ranging between 1 and 2000 . We note that if the length of one side of the triangle $2 \sqrt{3}$ was used in nondimensionalization, as it was used by Erturk and Gokcol [11], then our Reynolds number of 2000 would $2 \sqrt{3}$-fold such that it would correspond to a Reynolds number of 6928.

4.1. Equilateral Triangular Cavity. Let us first consider a nondimensional equilateral triangle with coordinates of corner points

$$
x_{a}=-\sqrt{3}, \quad x_{b}=\sqrt{3}, \quad h=1, \quad x_{c}=0, \quad y_{c}=-2,
$$

which was also considered by McQuain et al. [6], Jyotsna and Vanka [7], Li and Tang [8], Gaskell et al. [9], and Ribbens et al. [10]. We solved the flow in this triangle cavity at various Reynolds numbers ranging between 1 and 2000.

In order to verify the accuracy of the present numerical study, we consider arbitrary triangular cavity flow with the following analytical solutions [15]:

$$
\psi=\frac{y-x}{\operatorname{Re}}-e^{x+y}, \quad \omega=2 e^{x+y}
$$

For this model problem, as the boundary conditions we decided to use the analytical solutions defined in (43) at 


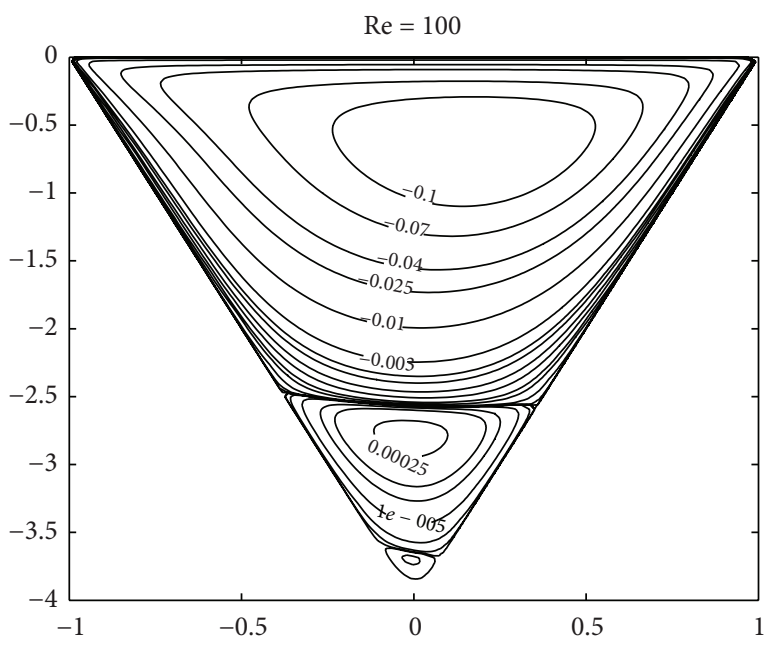

(a)

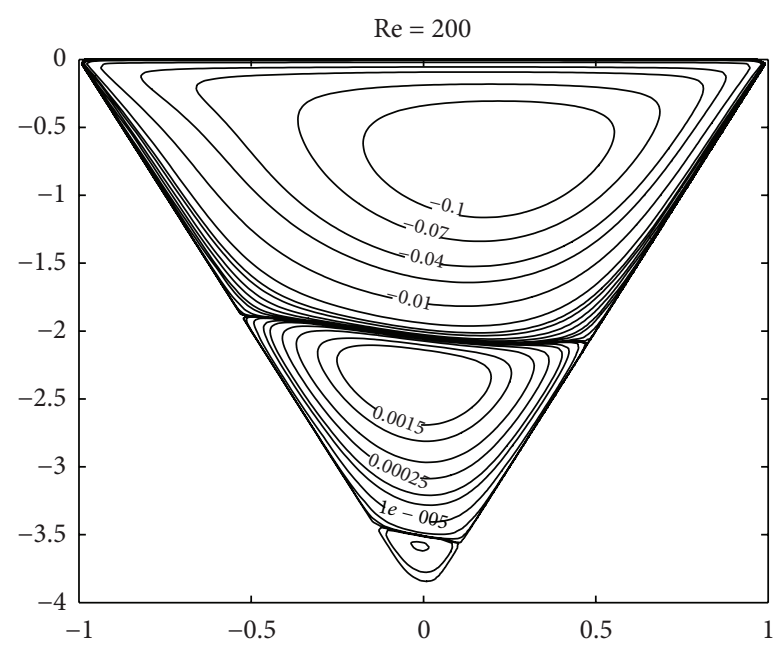

(b)

FIGURE 7: Contour figures of isosceles triangle.

the grid points on the boundaries. This way we would be able to avoid any effect of numerical boundary condition approximations on the numerical solution and concentrate on the accuracy of the solution of both formulations in the interior domain for given analytical values at the boundaries. We note that in (43), the vorticity $\omega$ is independent of Re and the solution of the streamfunction $\psi$ at different Reynolds numbers looks almost the same in a contour plot. We solve this model problem using different grid meshes, $11 \times 11 / 2$, $21 \times 21 / 2,41 \times 41 / 2,81 \times 81 / 2$, respectively. In these higherorder solutions, the average absolute $\left(E_{A A}\right)$ error and the the maximum norm $\left(E_{\mathrm{MAX}}\right)$ error between the exact solution $\mathrm{Exa}_{k}$ given in (43) and the numerical solution $\mathrm{Sol}_{k}$ obtained from (23) and (25) are defined as

$$
E_{A A}=\frac{1}{S} \sum_{k}\left|\operatorname{Exa}_{k}-\operatorname{Sol}_{k}\right|, \quad E_{\mathrm{MAX}}=\max _{k}\left|\operatorname{Exa}_{k}-\operatorname{Sol}_{k}\right|,
$$

where $S$ being the total number of grid points. Since the grid size is decreased by a factor of 2 , we can calculate the convergence rate $m$ using the following formula [16]:

$$
m=\frac{\log \left(E_{\Delta h} / E_{\Delta h / 2}\right)}{\log 2} .
$$

As observed by Wan and Zhou [17], the accuracy of the present results is getting better as the grid number increases, and we can see that when the grid spacing is decreased progressively by half, the scheme maintains fourth-order of spatial accurate; the convergence rate is very close to $m \approx$ 4. In Figure 2, the average absolute error $E_{A A}$ and the the maximum norm error $E_{\mathrm{MAX}}$ are plotted with respect to the grid spacing in a log-log scale [16]. From Figure 2, we can again clearly see that the fully HOC formulation indeed provide fourth-order accurate solutions; the slope between $\log E$ and $\log h$ is close to $m \approx 4$.
Figures 3 and 4 show the streamline and vorticity contours of the triangular cavity flow for a variety of Reynolds numbers obtained with using $(128 \times 128) / 2$ grids points. These figures show the streams and vortices that as the Reynolds number increases. From these contour figures, we conclude that the fourth-order compact formulation provides very smooth solutions and it is seen that as Re becomes large, the location of the center of the primary eddy and its streamfunction value seem to have converged. In terms of quantitative analysis, Table 1 tabulates the center of the primary eddy and the streamfunction and vorticity values at the core, together with results found in the literature. Our results are in good agreement with the results in [6] and [9] up to the maximum Reynolds number $(\operatorname{Re}=500)$.

In Figure 5(a), we plot the vorticity values at the center of the primary vortex tabulated in Table 1, with respect to Reynolds number. The results of $\mathrm{Li}$ and Tang [8] start to behave differently starting from $\mathrm{Re}=100$ from the rest of the results. Also in Figure 5(a), the results of McQuain et al. [6] start to deviate from present results and Gaskell et al.s [9] results after $\operatorname{Re}=200$. Having a different behavior, the vorticity value of McQuain et al. [6] shows an increase such that their vorticity value at $\mathrm{Re}=500$ is greater than the vorticity value at $\mathrm{Re}=350$, whereas the present results and Erturk et al's [11] results show a continuous decrease until $\operatorname{Re} \leq 1500$. We believe that these behaviors are due to the coarse grids used in those studies and in order to resolve these behaviors we decided to solve the same flow problem using several coarse grid meshes. For a given grid mesh we have solved the flow for increasing Reynolds number until a particular Re, where the solution was not convergent but oscillating. For this particular Re when the number of grid points was increased, the convergence was recovered and we were able to obtain a solution.

According to the mean square law [18], the value of vorticity is approximately constant at the primary vortex. For an equilateral cavity with length of side $2 \sqrt{3}$ this constant is 


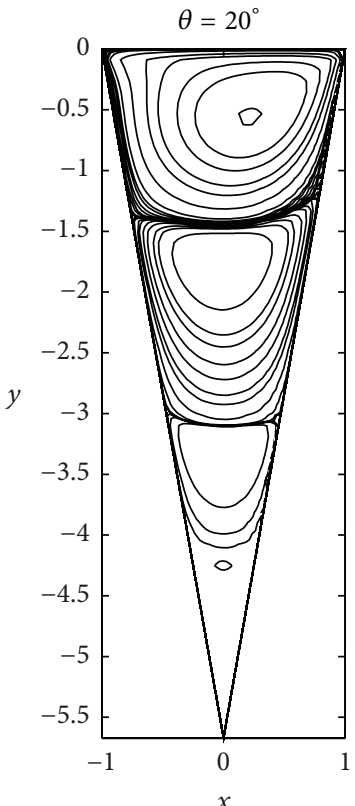

(a)

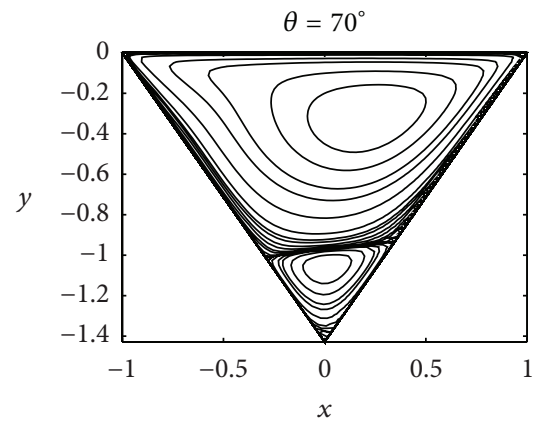

(c)

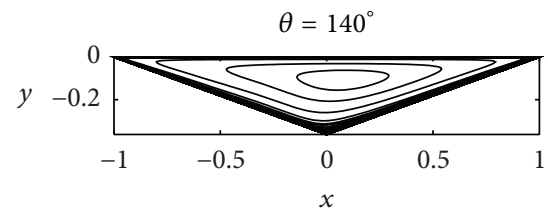

(e)

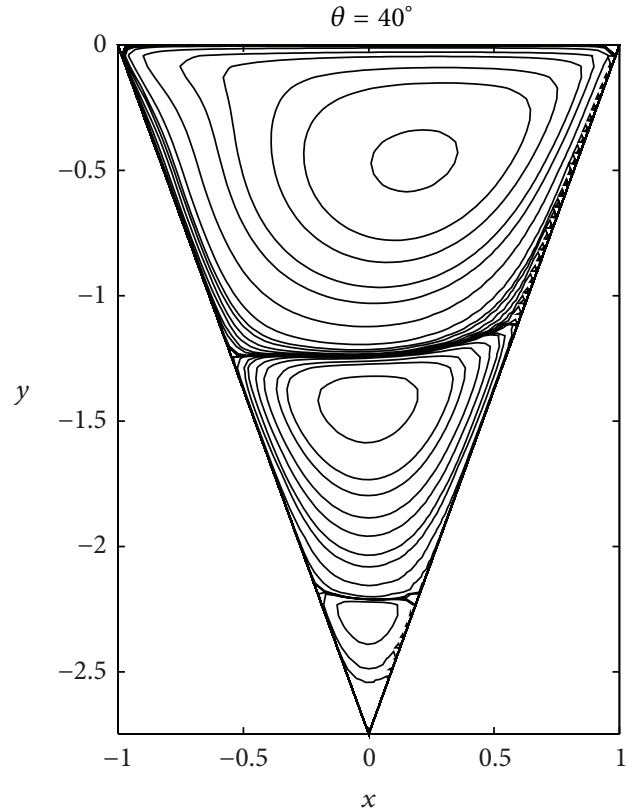

(b)

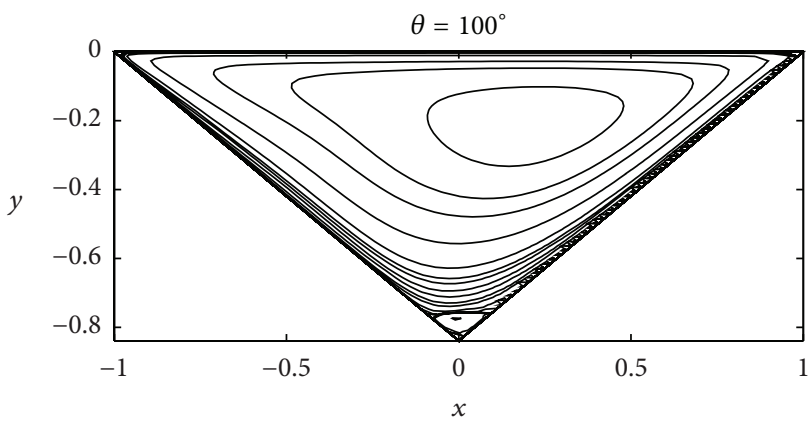

(d)

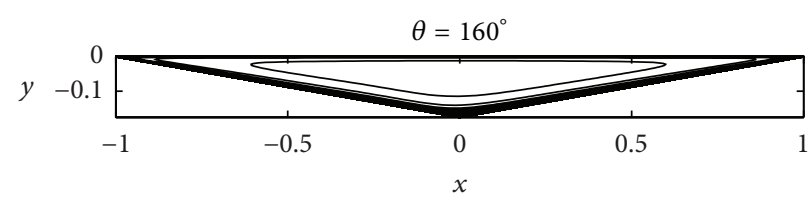

(f)

FIGURE 8: Contour figures of isosceles triangle with various corner angle.

found to be 1.054 by McQuain et al. [6]. This infinite Reynolds number value of the vorticity of 1.054 is shown with the dotted line in Figure 5(a). We note that especially the eddies at the bottom corner occupy some portion of the corner as Reynolds number increases. However, the increase in the size of the portion of the bottom corner eddies almost stops after $\mathrm{Re}=500$ and the size of the primary eddy remains almost constant beyond and so is the value of the vorticity at the core of the primary eddy. It looks like, for an equilateral triangular cavity flow at high Reynolds numbers, the mean square law predicts the strength of the primary eddy within an error due to the effect of the secondary eddies. For circular or elliptic boundaries Ribbens et al. [10], for square cavity flow Erturk and Gokcol [11], and for rectangle cavities McQuain et al. [6] have shown that the mean square law is approximately valid and successful in predicting the strength of the primary eddy at high Reynolds numbers.

4.2. Scalene Triangle Cavity. Owing to the unsymmetric geometry, flow motion in a scalene triangular cavity is very difficult to simulate. It was conjectured in McQuain et al. [6] and Ribbens et al. [10] that it might be unavoidably ill-conditioned. Then we considered a scalene triangle with coordinates of corner points

$$
\begin{gathered}
x_{a}=-\sqrt{3}, \quad x_{b}=\sqrt{3}, \quad h=1, \\
x_{c}=\frac{\sqrt{3}}{2}, \quad y_{c}=-2 .
\end{gathered}
$$




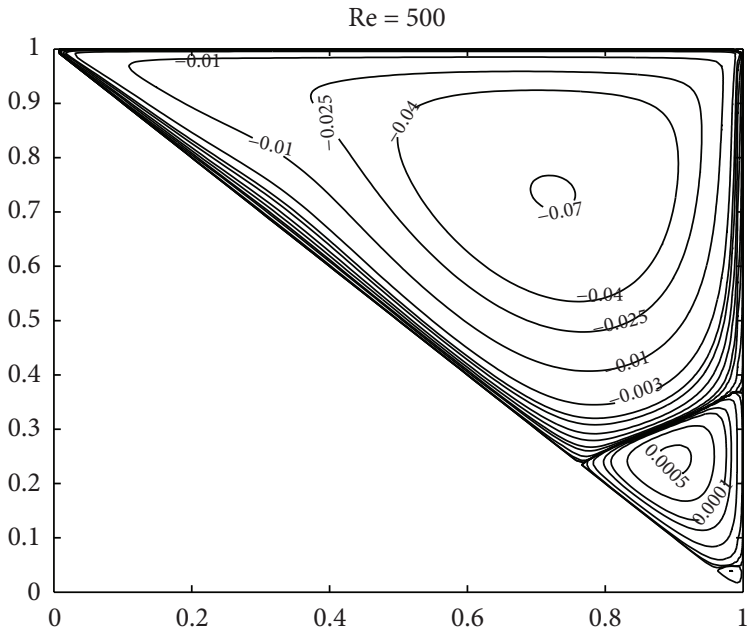

(a)

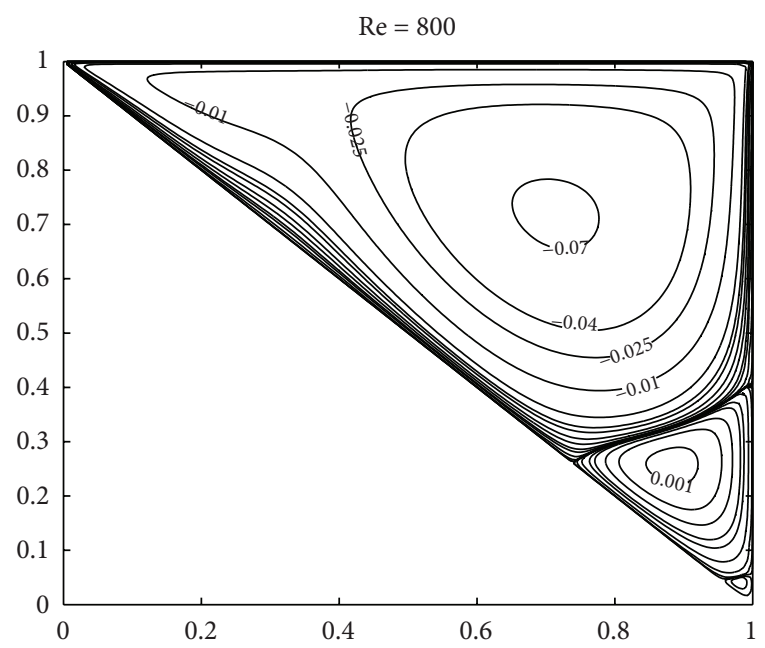

(b)

FIGURE 9: Contour figures of right hand side aligned right triangle at different Reynolds numbers.

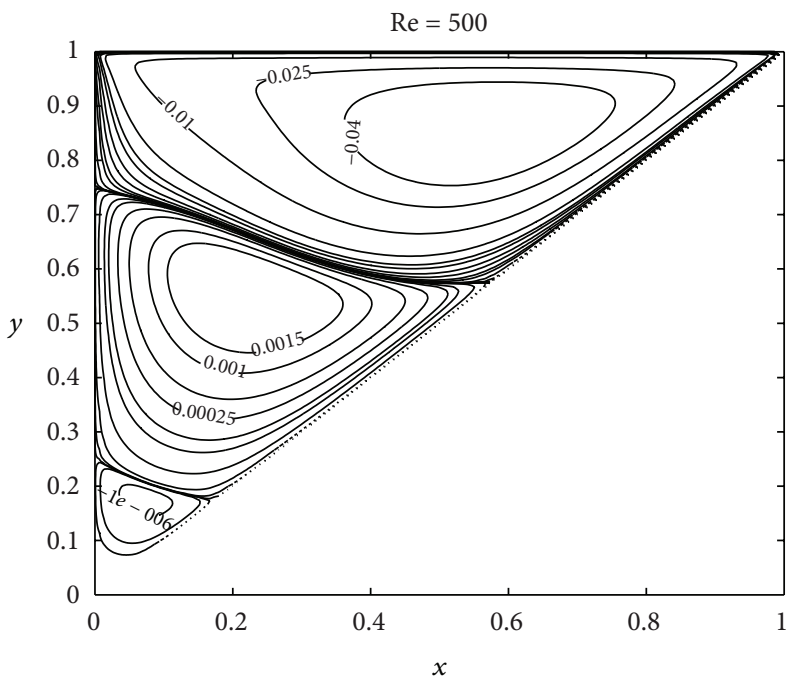

(a)

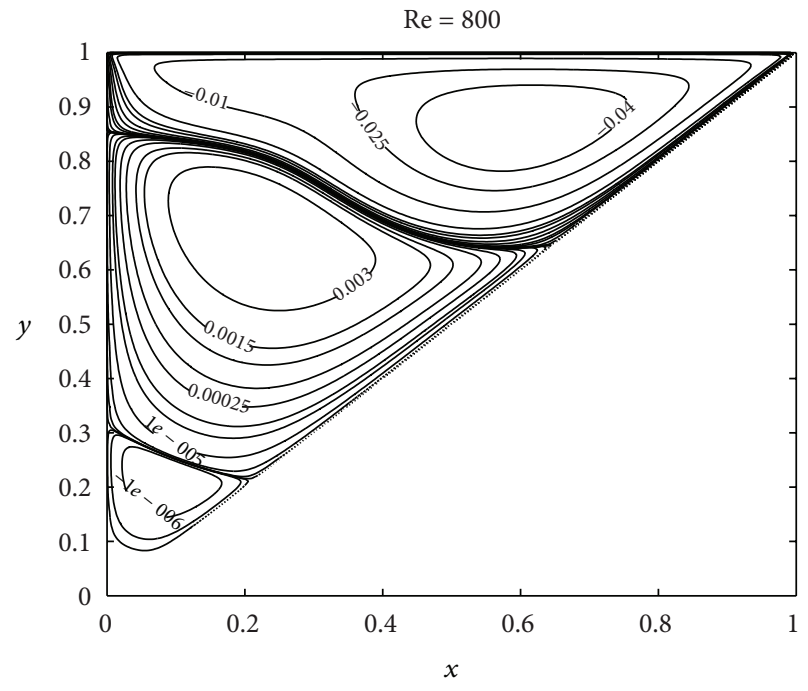

(b)

FIGURE 10: Contour figures of left hand side aligned right triangle at various Reynolds numbers.

Using this triangle geometry, we could carry out the calculations for Reynolds numbers as high as 800. In Figure 5(b), we plot the vorticity values at the center of the primary vortex obtained with present study and Li and Tang. [8]. We can see from Figure 5(b) that the vorticity values are getting more and more close to the theoretical value as the Reynolds number increases. In Figure 6, we plot the streamlines and vorticity contours for $\operatorname{Re}=350$ and 500. Again it is seen that as $\mathrm{Re}$ becomes large, the location of the center of the primary eddy and its streamfunction value seem to have converged.

4.3. Isosceles Triangle Cavity. We first considered an isosceles triangle which was also considered by Jyotsna and Vanka [7], Gaskell et al. [9], and Erturk and Gokcol [11], where

$$
x_{a}=-1, \quad x_{b}=1, \quad h=0, \quad x_{c}=0, \quad y_{c}=-4 .
$$

We note that our definition of Reynolds number is equivalent to one fourth of the Reynolds number definition used by Jyotsna and Vanka [7]. Figure 7 shows the streamline contours of the flow in this triangle at various Reynolds numbers. Comparing the location of the primary eddy center with that of Jyotsna and Vanka [7], Gaskell et al. [9], and Erturk and Gokcol [11], we believe that our results are more accurate.

Then we considered an isosceles triangle which was also considered by Gaskell et al. [9] and Erturk and Gokcol. [11], with

$$
x_{a}=-1, \quad x_{b}=1, \quad h=0, \quad x_{c}=0, \quad y_{c}=-\cot \frac{\theta}{2},
$$

where $\theta=5^{\circ}, 10^{\circ}, 20^{\circ}, 40^{\circ}, 70^{\circ}, 100^{\circ}, 140^{\circ}, 160^{\circ}$, and $170^{\circ}$. Figure 8 qualitatively shows the change in the flow with $\mathrm{Re}=$ 200 in an isosceles triangle as the corner angle, $\theta$, changes. 
We also considered an isosceles right triangle with the $90^{\circ}$ corner being at the top right corner, such that with corner points [11]

$$
x_{a}=0, \quad x_{b}=1, \quad h=1, \quad x_{c}=1, \quad y_{c}=0 .
$$

Figure 9 shows the flow topology as a function of Reynolds numbers and at last, we also considered an isosceles right triangle with the $90^{\circ}$ corner being at the top left corner, such that with corner points [11]

$$
x_{a}=0, \quad x_{b}=1, \quad h=1, \quad x_{c}=0, \quad y_{c}=0 .
$$

Using this triangle geometry, we were able to obtain the solutions and Figure 10 shows the flow topology as a function of Reynolds numbers. For the considered triangle geometry, we can see that the eddy is closest to the moving lid. As it is obvious from these figures, in both cases, the flow behaves very differently as Reynolds number increases, which shows that the flow structures in a triangle cavity are greatly affected by the triangle geometry.

\section{Conclusion}

In this work we have developed a new semicompact fourthorder scheme for the time-independent $\psi$ - $\omega$ form of the 2D, incompressible N-S equations governing the fluid flow in a triangular driven cavity. Our numerical scheme has been proved robust for a wide range of Reynolds numbers and applicable to triangular cavities with arbitrary shape. The key point with the present scheme is that it allows direct iteration for low-to-high Reynolds numbers. It is the success of the current method with the wide range of $\mathrm{Re}$ and mesh sizes that indicates the potential of this method as an accurate and stable numerical method applicable to a wide range of problems. We have tested the present method for both the equilateral triangular cavity problem and the scalene triangular cavity problem, and excellent agreement is found in all the cases, both qualitatively and quantitatively.

\section{Conflict of Interests}

The authors declare that there is no conflict of interests regarding the publication of this paper.

\section{Acknowledgment}

This work is supported by the National Natural Science Foundation of China (no. U1304106).

\section{References}

[1] E. Barragy and G. F. Carey, "Stream function-vorticity driven cavity solution using p finite elements," Computers and Fluids, vol. 26, no. 5, pp. 453-468, 1997.

[2] Z. F. Tian and Y. B. Ge, "A fourth-order compact finite difference scheme for the steady stream function-vorticity formulation of the Navier-Stokes/Boussinesq equations," International Journal for Numerical Methods in Fluids, vol. 41, no. 5, pp. 495-518, 2003.
[3] J. C. Kalita, A. K. Dass, and D. C. Dalal, "A transformation-free HOC scheme for steady convection-diffusion on non-uniform grids," International Journal for Numerical Methods in Fluids, vol. 44, no. 1, pp. 33-53, 2004.

[4] X. H. Wu, J. Z. Wu, and J. M. Wu, "Effective vorticity-velocity formulations for three-dimensional incompressible viscous flows," Journal of Computational Physics, vol. 122, no. 1, pp. 6882, 1995.

[5] M. Li, Numerical solutions for the incompressible NavierStokes equations [Ph.D. thesis], Simon Fraser University, British Columbia, Canada, 1998.

[6] W. D. McQuain, C. J. Ribbens, C. -Y. Wang, and L. T. Watson, "Steady viscous flow in a trapezoidal cavity," Computers \& Fluids, vol. 112, no. 1, pp. 173-181, 1994.

[7] R. Jyotsna and S. P. Vanka, "Multigrid calculation of steady, viscous flow in a triangular cavity," Journal of Computational Physics, vol. 122, no. 1, pp. 107-117, 1995.

[8] M. Li and T. Tang, "Steady viscous flow in a triangular cavity by efficient numerical techniques," Applied Mathematics and Computation, vol. 32, pp. 55-65, 1996.

[9] P. H. Gaskell, H. M. Thompson, and M. D. Savage, "A finite element analysis of steady viscous flow in triangular cavities," Journal of Mechanical Engineering Science, vol. 213, no. 3, pp. 263-276, 1999.

[10] C. J. Ribbens, L. T. Watson, and C. -Y. Wang, "Steady viscous flow in a triangular cavity," Journal of Computational Physics, vol. 112, no. 1, pp. 173-181, 1994.

[11] E. Erturk and O. Gokcol, "Fine grid numerical solutions of triangular cavity flow," Applied Physics, vol. 38, no. 1, pp. 97-105, 2007.

[12] G. D. Simth, Numerical Solution of Partial Differential Equations, Oxford University Press, Oxford, UK, 2nd edition, 1984.

[13] Y. V. S. S. Sanyasiraju and V. Manjula, "Higher order semi compact scheme to solve transient incompressible NavierStokes equations," Computational Mechanics, vol. 35, no. 6, pp. 441-448, 2005

[14] W. F. Spotz and G. F. Carey, "High-order compact scheme for the steady stream-function vorticity equations," International Journal for Numerical Methods in Engineering, vol. 38, no. 20, pp. 3497-3512, 1995.

[15] C. W. Richards and C. M. Crane, "The accuracy of finite difference schemes for the numerical solution of the NavierStokes equations," Applied Mathematical Modelling, vol. 3, no. 3, pp. 205-211, 1979.

[16] J. Breil and P.-H. Maire, "A cell-centered diffusion scheme on two-dimensional unstructured meshes," Journal of Computational Physics, vol. 224, no. 2, pp. 785-823, 2007.

[17] D. C. Wan and Y. C. Zhou, "Numerical solution of incompressible flows by discrete singular," International Journal for Numerical Methods in Fluids, vol. 38, pp. 789-810, 2002.

[18] G. K. Batchelor, An Introduction to Fluid Dynamics, Cambridge University Press, Cambridge, UK, 1967. 


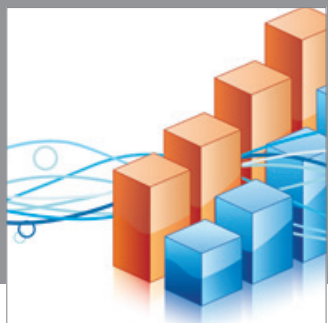

Advances in

Operations Research

mansans

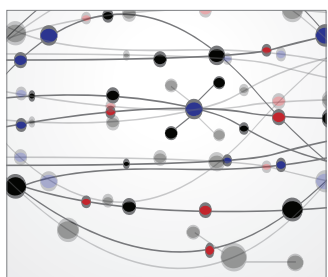

The Scientific World Journal
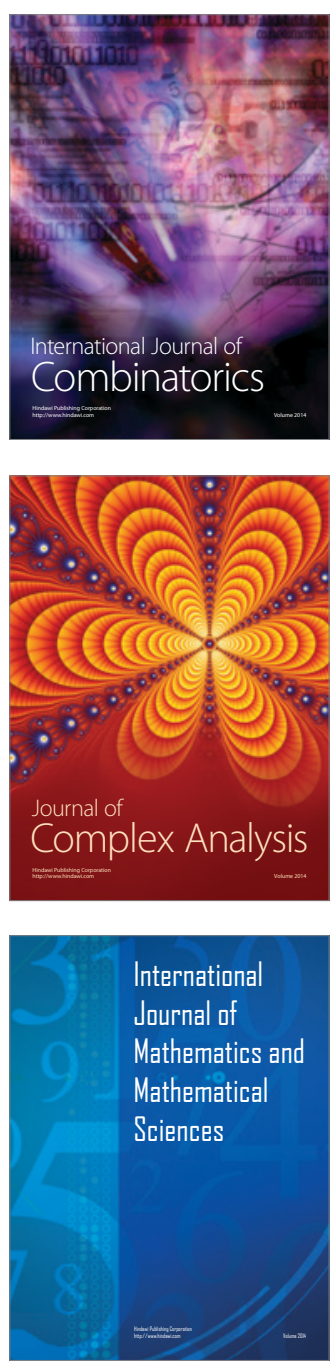
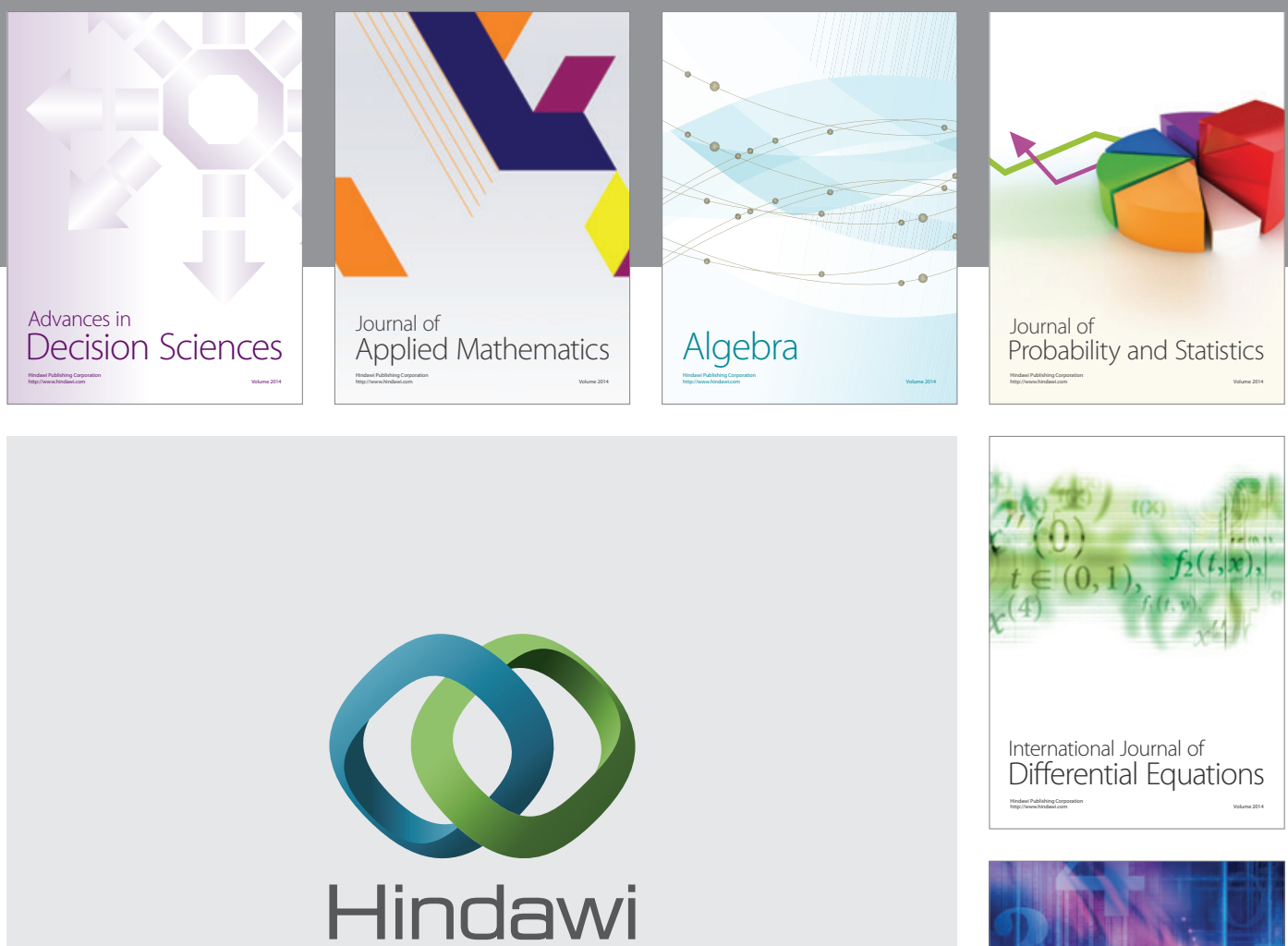

Submit your manuscripts at http://www.hindawi.com
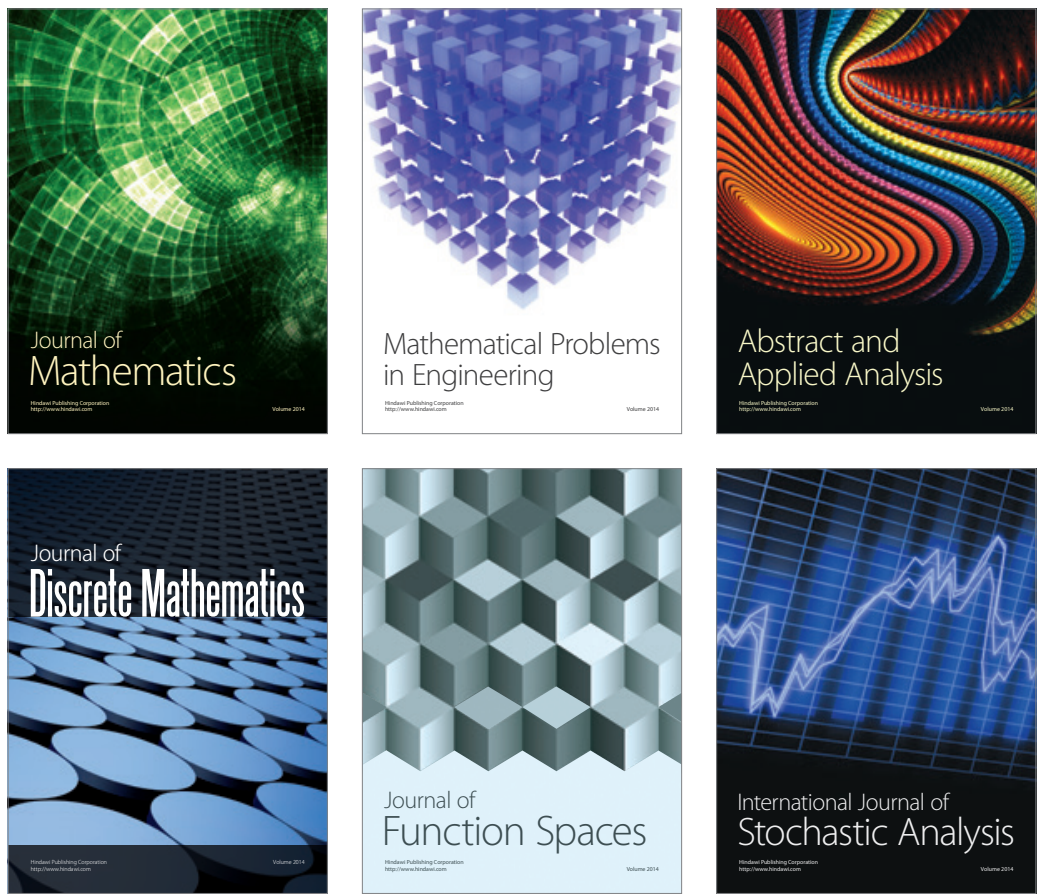

Journal of

Function Spaces

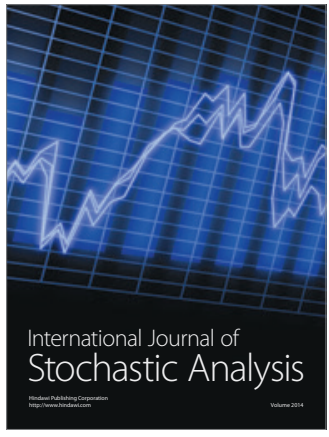

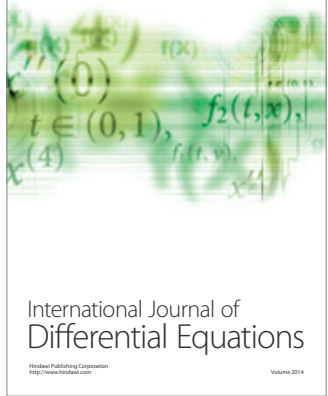
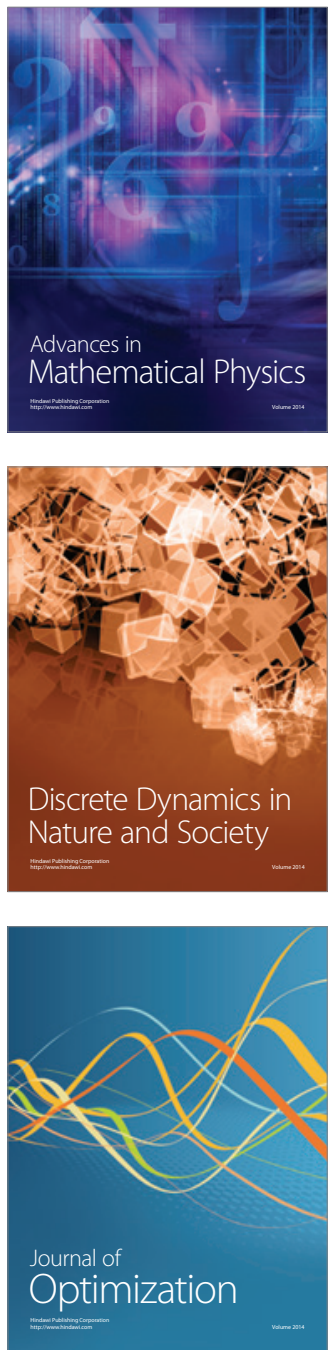